\title{
Designing and Doing Field Experiments ${ }^{1}$
}

Tobias Wolbring, Chair of Empirical Economic Sociology, FAU Erlangen-Nürnberg

\section{Introduction}

Experiments can be conducted in different settings in principle. In the previous chapter, we introduced the laboratory experiment, which is staged in the rather sterile, anonymous, and controlled setting of a laboratory. Most people have this research design in mind when talking about experiments. However, other variants of the experiment exist. A second important group of experiments are so-called field experiments. Field experiments rely on the three design elements common to all experiments - manipulation, group comparison, and randomization and, in addition, are staged in a "natural" research setting where individuals usually interact in everyday life and regularly complete the task under investigation. This feature of "naturalness" is thus the core feature distinguishing field experiments from experiments in other settings. It is also one of the major reasons why researchers use field experiments: They allow the investigation of social behaviour under "natural" conditions. This becomes obvious in the following example:

Imagine the following situation: In the 1960ies, two luxury and two rusty old cars repeatedly blocked different intersections in Palo Alto and Menlo Park, California, although the traffic lights turned green. After a few seconds, most drivers waiting behind the car began to honk, often more than once signalling their anger about the unnecessary delay. The type of car luxury vs rusty old - blocking the way was the manipulated treatment in this experiment by Dobb and Gross (1968). Drivers were randomly assigned to one of the two treatment conditions; their honking behaviour was recorded and compared across the two experimental groups

While one might suspect that this experience is similarly upsetting for the affected drivers irrespective of who caused it, Dobb and Gross (1968) showed in the sketched field experiment that human behaviour is substantially influenced by the perceived social status of others: 84 percent honked the horn in case a low status car blocked the way, but only 50 percent did so if a luxury car caused the delay. The study reveals that individuals with a high status are treated more preferable than those with a low status in this and other domains of social life (for a review see Ridgeway 2014). While many people would probably not admit such differential treatment of others in surveys or are not even aware of this latent mechanism steering our behaviour and

\footnotetext{
${ }^{1}$ This is a preliminary draft of a chapter originally planned for a textbook on "Designing and Doing Experiments". I am grateful to Alysha Owen, Stefanie Eifler, and Marc Keuschnigg for helpful input and hope that the text turns out useful for anyone who is planning to conduct an experiment or who is teaching a class on experimental methods.
} 
while it is hard to imagine a lab experiments on this specific topic, concealed field experiments can help to uncover this fact of social life.

Field experiments like this have a long tradition in some areas of the social and behavioural sciences and have become have become increasingly popular in others (for reviews see Baldassari and Abascal 2017; Gneezy 2017; John 2017; Levitt/List 2009). Besides the wellknown strengths of experimental designs for causal inference, a major asset of field experiments is that they allow to bring social context back in. Hence, as compared to the "sterility of the laboratory environment" (Harrison and List 2004: 1010) field experiments help to attenuate concerns about realism and external validity.

For example, in the horn-honking experiment by Dobb and Gross few doubts remain whether these findings can be transported to other areas of the U.S. of that time. As more recent replications and extensions of the original experiment have demonstrated, the findings even extend to other time periods, countries, and behavioural measures, although the exact status signals themselves might vary with the specific time period and situation (for replications and extensions of the original experiment see Diekmann et al. 1996; Jann 2009; Wolbring 2013). Bringing a realistic social context into experiments and allowing to make generalizations under weaker assumptions is thus one of the key advantages of field experiments providing a rationale why they deserve a closer look.

In this chapter, we explain in more detail what it actually means to conduct an experiment under "natural" conditions. We thereby recur to Cronbach's (1982) concept of utos to emphasize that naturalness encompasses not only the experimental setting (s), but also the ,units of study $(\mathrm{u})^{6}$, ,treatment of the research design $(\mathrm{t})^{6}$, and ,the measurement of outcomes $(\mathrm{o})^{6}$. Gains in naturalness often come at the cost of some loss in field control. Thus, we then elaborate on methodological pitfalls of field studies, i.e. problems resulting from limited field control which refer to the three core features of experiments. The chapter concludes with a discussion of ethical, political, and practical challenges of conducting experiments in the field.

\section{Field experiments as experiments under natural conditions}

As highlighted in the introduction to this chapter, "naturalness" encompasses the social context in a rather broad sense and is not limited to the experimental setting. Researchers care about naturalness, because it can influence the external validity and generalizability of experimental results. However, it is important to note that it is not necessary to accurately map all aspects of social life in an experiment for externally valid results. A close match with the "real" world is only essential for those aspects which might have an influence on the direction and strength of 
the treatment effect - technically speaking: an interaction of the treatment effect with the specific social environment. For example, it might be the case that the same individuals are more cooperative in the lab than in the "real" world and hence react differently to a stimulus in the two environments. In such a case, it is important to conduct the study in an environment that matches the social context of the event you are trying to study and does not contain any noticable unrealistic elements.

To get a better sense of what a realistic social context entails, it is useful distinguish between different dimensions. Building on Cronbach's (1982) utos framework on external validity, we distinguish four dimensions of naturalness: ${ }^{2}$

Units: A field experiment scores high on this dimension of naturalness, if the study is based on a group of subjects that actually completes the tasks under investigation in the "real" world or is the target of the intervention to be evaluated. If this is not the case, the assumption becomes essential for generalization that the actual population of interest would have reacted in a similar way to the treatments as the experimental units have. This assumption of no interaction between treatment effect and population might hold in certain studies, but as long as it remains untested it is a source of uncertainty and might - in case of violation - lead to wrong inferences and generalizations. Researchers should thus aim to conduct the study with the actual population of interest. For example, in a field experiment on cheating behaviour in finance, one would aim to recruit a sample of professional bankers rather than students or people from the general population, because the former have more knowledge about and more experience with finance and probably apply different decision heuristics than the latter (for an experiment on cheating with bank employees see Cohn, Fehr, and Maréchal 2014).

Treatments: The treatment in a field experiment should be implemented and administered in a way that closely mirrors the intervention as it might have happened without a scientific study exploring its causal effects. So for example, if a foundation is planning a large-scale vaccination program and commissions a small-scale evaluation study to determine the impact of the intervention, researchers trained in causal inference will seek to achieve maximum similarity between the field experiment and the planned large-scale intervention regarding the form of the treatment, the staff administering the vaccination, and the exact implementation procedure. For that reason, conducting field experiments often involves

\footnotetext{
${ }^{2}$ We follow the reading of Shadish et al. (2002) of Cronbach's typology. However, it is worth noting that Cronbach (1982) introduced further distinctions between utos, UTOS, and *UTOS which we do not pick up in this textbook for the sake simplicity.
} 
intensive cooperation and coordination with social agents and local partners responsible for the implementation of the treatment in practice. Moreover, subject's knowledge of ongoing research might limit the degree of naturalness by changing their reactions to the treatment. Hence, within the bounds of ethics and law, it is advisable from a causal inference perspective to reveal only as much as necessary about the study and its research questions to the subjects and the persons implementing the treatments.

Outcomes: The recommendation in the previous paragraph is closely linked to the measurement of outcomes. Thereby, one can distinguish between potentially reactive measures on the one hand and unobtrusive modes of data collection on the other. While potentially reactive measures, by definition, can cause unnatural reactions of the subjects, unobtrusive modes of measurement remain undetected by the persons under investigation. As a consequence, unobtrusive modes of data collection, like those employed in the horn-honking experiment by Dobb and Gross (1968), usually offer a higher degree of naturalness and are, ceteris paribus, preferable from a methodological perspective. At the same time, researchers also need to answer further questions regarding measurement such as operationalization of core theoretical construct, frequency of measurement, and optimal timing of measurement. Especially if the treatment has effects on multiple outcomes and if effect strengths are not constant over time, these decisions - as choices of the outcome measure -can substantially influence the findings of a field experiment.

Setting: This fourth dimension of naturalness focuses on the location of the experiment, the field in the classical sense of the word. The setting should be as realistic as possible; in the optimal case the experiment is staged at the location where the tasks and actions of interest usually take place in the "real" world. The call for a natural location additionally implies that besides the implementation of the treatment and the measurement of outcomes changes of the setting should be kept to a minimum. This concerns both physical conditions such as the placement of furniture, technical devices etc. as well as the social environment such as the presence of individuals, their social roles in the situation at hand, and incentives for action. Changing the original setting of the experiment too much (for example, by modifying commodities, tasks, and stakes) might interrupt everyday routines and lead to behavioural changes not caused by the treatment itself undermining inference. For example, in a study on stealing in break rooms, the presence of a camera for measuring the outcome will - if detected by the subjects - heavily influence the observed extent of stealing regardless of the effectiveness of the treatment (for the crime preventing effects of CCTV see Piza 2018). 
In order to design a realistic field experiment that closely mirrors the "real" world one should aim to score high on all four dimensions on "naturalness" (see Table 1 for an overview). Thereby, those factors that potentially interact with the treatment effect deserve special attention, as they are crucial for a high degree of external validity. Our analytical distinction between four different dimensions of "naturalness" further illustrates that naturalness is not an experiment quality that is either present or not; it is assessed in degrees. A field experiment is realistic with respect to certain dimensions and the degree of naturalness can be located on a multidimensional space regarding the four continuous axes 'units', 'treatments', 'outcome', and 'setting'.

Some combinations rarely occur in applied research. For example, it is hard to imagine a field experiment which is staged in a realistic setting (e.g., a local market), but relies on a sample of unnatural experimental units (without visitors of the market). Since the dimensions are not completely independent from each other, the analytical distinction is somewhat blurry and not always as clear cut in practice as it appears in theory. Nonetheless, distinguishing between them is useful, since it raises awareness for the different aspects of external validity and helps to identify potential problems when designing the experiment (see also section 3).

Furthermore, the question arises whether an experiment that scores high on one dimension of "naturalness", but not on the remaining three can actually be considered as a field experiment. For example, Gächter and Herrmann (2011) invited rural residents of Russian villages in the lab to study behaviour in a public good game. Without any question, this design scores high on the dimension 'units', while the other design elements suggest a classical lab experiment. Harrison and List (2004) propose to call such designs with nonstandard subject pools "artefactual field experiments". In contrast, so-called "lab-in-the field experiments" do not only cover nonstandard subject pools, but bring the laboratory to the field without actually staging it in the natural setting of the subjects.

So are studies like these already field experiments? The literature disagrees on this point. While some, like Harrison and List (2004), seem to perceive these designs as a special variant of field experiments (calling them artefactual and lab-in-the field experiments), we argue that they are still lab experiments, because they take place in the rather sterile, abstract, and controlled lab environment and not in the natural setting of the subjects. In our viewpoint, the dimension 'setting' hence gains special prominence in the definition of a field experiment (see subsection 1) and we propose to use the term "field experiment" only, when a study is actually conducted in the field, e.g. a "natural" setting where individuals usually interact in everyday life and regularly complete the task under investigation. Due to this feature of field experiment, 
the degree of control over the social context is usually limited in experiment in this setting and lower than in lab experiments with tailor-made artificial environments. This has important ramifications for potential methodological pitfalls which deserve special attention in field experiments.

\section{Pitfalls of Experimenting in the Field}

Gains in the degree of naturalness in field experiments often come at the prize of a certain loss in field control. As compared to lab experiments, field experiments offer a less controlled environment and are, thus, more prone to certain pitfalls which can result in deviations from the experimental ideal regarding all three core design elements - treatment, group comparison, and randomization - undermining causal inference. Knowing those potential caveats is useful and helps to avoid them when designing and doing experiments.

\subsection{Caveats Concerning the Treatment}

In this subsection, we discuss problems related to the manipulation of the treatment variable. The presentation will concentrate on issues of reduced treatment reliability and validity and will highlight the potential contribution of manipulation checks and other practical measures to address these problems.

For practical reasons, interventions in field settings are usually not implemented by researchers themselves, but by third parties. These third parties frequently are students supporting a research project or staff of local cooperation partners having better access to the field. Obviously, the very fact that researchers have to rely on such other agents for treatment implementation in the field increases the risk that the intervention is not implemented as originally intended. This can affect both the so-called treatment reliability and treatment validity.

Limitations of treatment reliability occur if different staff members implement the same treatment differently. As a consequence, the experiment does not inform about the treatment as originally intended, but about the average effect of a variety different variants of the treatment. An increase in treatment heterogeneity reduces statistical power of the analysis and, more importantly, can lead to biased results in case of substantial implementation heterogeneity (see also next paragraph on treatment validity). For illustrative purposes, imagine a rather extreme scenario where half of the agents implements an effective treatment, while the other half implements a totally ineffective intervention. Apparently, estimates of the average treatment 
effect will mix the effective and ineffective treatment. Based on such a result policy-makers might decide not to adopt the intervention on large-scale because it is not sufficiently effective.

As this example illustrates, measures to ensure treatment reliability are highly recommended. At first place, a thorough briefing and training of the staff implementing and administrating the treatment is essential. Procedures for implementation should be as standardized as possible and the degrees of freedom during treatment implementation should be kept to a minimum. Written instructions for the experimental staff can help to secure a high quality and consistency of implementation. In addition, researchers might want to go to the field themselves from time to time to monitor for randomly drawn samples how the treatment is actually implemented. Monitoring can help to uncover problems and allow to intervene early enough to fix the problem before it bungles the whole experimental design.

Limited field control and reliance on third parties can not only result in low treatment reliability, but also in limitations of treatment validity. Treatment validity is high if the intervention is implemented as originally intended. Treatment reliability is thereby a necessary but not a sufficient precondition for treatment validity. That means if treatment reliability is low, then the treatment cannot be valid. However, high treatment reliability does not automatically imply high treatment validity. For example, different students can always implement the treatment in a consistent way. Nonetheless, this reliable implementation of the treatment might be flawed. Such implementation errors can occur for different reasons. Misunderstandings or mistakes might accidentally lead to deviations from the original treatment. However, third agents might also deviate from the research plan on purpose. Especially, if staff members have a strong opinion about the topic of the field experiment and have an interest in a certain outcome of the study, they might try to influence the results of the experiment by modifying the treatment or not administrating it at all.

Besides field monitoring, manipulation checks provide additional help to secure treatment validity. As in other types of experiments, for the intervention to have a realistic chance to be effective it is important to ensure that subjects get treated in the sense that they actually receive the treatment and are aware of it. In field experiments this is particularly important, because subjects in real-world settings might focus on other aspects of the decision situation than the stimulus. For example, one might try to experimentally influence car drivers with an information campaign warning about the risks of speeding, but they might perceive it as just another type of advertisement. Apparently, an important precondition for this intervention to be effective is that the drivers actually receive the information material, read the content, and change their subjective evaluation of risks caused by speeding. It is thus important to conduct 
pretests and to explore in manipulation checks whether these assumptions actually hold. In addition to pilot studies, researchers can also conduct such manipulation checks with the data from the actual experiment by testing whether the treatment not only affects the outcome of interest but also certain theoretically expected intervening variables.

At the same time subjects' knowledge of being part of an experiment can also cause unwanted behavioural changes. While it is essential for a high treatment validity that subjects are actually aware of the intervention, it is a thin line between securing sufficient awareness and causing unwanted reactivity. Similar to the lab, reactivity can occur in field experiments when experimental units are informed about the study and its topic. For example, subjects might start to behave in a socially desirable way, might try to prove or disprove the (actual or expected) research hypothesis, or might try to generate an outcome that supports a certain policy. A famous example is the Hawthorne effect (Gillespie 1991; Roethlisberger and Dickson 1939): Productivity of industrial working groups at Western Electric's factory at Hawthorne substantially increased merely due to the fact that workers became aware that scientists conducted a study with them. The presence of reactivity causes serious problems, because the experimental results loss both external and internal validity. Similar problems also arise if deviations from random sampling and random assignment occur.

\subsection{Caveats Concerning Group Comparisons}

Besides pitfalls regarding treatment manipulation further deviations from the experimental ideal can undermine the comparability of the experimental group. In this subsection, we discuss problems related to comparison of experimental groups. The presentation will first focus on interference between experimental units, then discuss the scalability and context-dependency of field experimental results, and finally reflect on measuring short- and long-term effects of treatments in the field.

One particularly pressing issue in field experiments can be interference between experimental units. Due to limited field control, researchers cannot always prevent that research units belonging to different experimental groups come in contact with and influence each other. In the course of such interactions, knowledge can spill over, social contagion can be at work, and positive or negative externalities of the treatment can affect the control group. For example, in the case of a vaccination program those not receiving the treatment might profit from a reduced risk of being infected due to a general equilibrium effect (positive externalities). In contrast, participation in a job training program might give applicants for a new job a head start over other unemployed not receiving the intervention resulting in a crowding out of the controls 
by the treated (negative externalities). Finally, subjects in the control group might become aware of the intervention due to the experiment and might search for alternative ways to get treated, fully undermining the comparison between experimental groups.

Technically speaking, interference between experimental groups leads to a violation of one of the fundamental preconditions for counterfactual causal inference: the stable unit treatment value assumption (SUTVA). Remember that the SUTVA requires - among other things - that there is "no interference between units (...) leading to different outcomes depending on the treatments other units received" (Rubin 1980: 591). The SUTVA is often unrealistic: Social life is full of examples where the conditions are not given. General equilibrium for example occur in the case of vaccination where it is sufficient to reach a certain threshold of vaccinated inhabitants to nearly completely eradicate a disease. And crowding out effects might occur, for example, for job training programs, because the program can increase the labour market chances of participants but can lower the chances of job-seekers competing with the trained applicants. Researchers can prevent some of these social processes leading to violations of the SUTVA by choosing smart experimental designs. For example, they might choose experimental locations for the treated and control with sufficient geographical and social distance, so that social interactions between units from different experimental groups become unlikely. Another way to avoid spillover effects and knowledge diffusion can be confidentiality requirements for the treated.

If interference is nonetheless unavoidable, one can either design an experiment that relaxes the SUTVA assumption or one can try to directly model spillover processes and to adjust for resulting biases in causal inference (for details see Gerber and Green 2012, Chap. 7). Obviously, this requires substantive knowledge about the social processes that cause the trouble. In addition, researchers need to be aware of the presence of interference in the first place to take such measures. It is thus important to keep a weather eye on the field during the field phase of the experiment asking for potentially problematic processes such as interaction between unit of different experimental groups.

This discussion of interference between groups also raises important questions about the scalability and context-dependency of experimental results, or in other words their generalizability regarding the utos dimensions 'treatment' and 'setting'. Researchers often conduct small-scale experiments in specific social contexts aiming to inform rather general and wide-ranging policy decisions. However, due to general equilibrium effects, crowding out, and social influence, it is far from clear whether the results of such experiments allow to draw inferences about the effectiveness of large-scale social programmes. As highlighted by the 
Nobel Prize winner Angus Deaton (2010; see also Deaton/Cartwright 2018), what works in the small scale might not work in the large scale and vice versa. At the same time, it remains unclear whether empirical findings can be transported to different conditions, since every study is based on specific units, treatments, observing operations, and social settings (Falk and Heckman 2009).

Cumulative research in the form of replication studies systematically varying the different utos dimensions provides answers about the context-dependency of experimental results. In addition, precise theorizing about the causal mechanisms driving treatment effects and specifying the conditions under which a particular treatment should be effective helps to address both issues of scalability and context-dependency (Lynch 1999). Thus, a stronger interlinkage of field experiments and social theory can help to mitigate concerns about generalizability, particularly if small-scale experiments are supposed to offer answers to "big" questions.

Finally, different temporal aspects should be kept in mind when designing and doing field experiments since they can influence the validity of experimental results. First, for financial or practical reasons, treatments are usually implemented for a certain time period only. If treatment effects vanish quickly after the end of the intervention, results can be quite misleading even though the study relies on an experimental design. Choosing the right timing between treatment and outcome measurement can be essential for identification of causal effects and valid conclusions about the effectiveness of a treatment. A second, related point is the identification of long-term effects of an intervention. While politicians are often short-term oriented wanting quick results on the effectiveness of a social program, it can take years and can be very costly for field experiments to provide answers about the long-term effects of social interventions. Especially, if repeated measurement of the same units over time is necessary, as is the case for studies aiming to identify long-term effects, a third and last point regarding time becomes obvious: Experimental subjects might drop out of the study due to death, might be untraceable due to moves, or might refuse to participate in further stages of data collection. As is well known from methodological research on panel data (e.g., Lynn 2009), these different types of attrition can introduce systematic bias. Further, attrition can also undermine random assignment if units in the treatment or in the control group differ in their propensity to drop out of the study. Establishing a good tracking system of subjects and providing incentives to further participate in the experiment are effective measures to minimize attrition. In addition, because information on these subjects from previous measurement is available, researchers can try to correct for related bias by means of multiple imputation and weighting (for introductions see Allison 2001; Enders 2010; Little and Rubin 2002). Although those corrections on statistical grounds are not 
free of assumptions about the sources of dropout, they can help to cure the problem and allow to quantify the degree of uncertainty introduced into the estimation of treatment effects by missing data.

\subsection{Caveats Concerning Random Sampling and Assignment}

In this subsection, we discuss problems related to random sampling from a population of interest and assignment of units to experimental groups. The presentation will concentrate on issues of subject recruitment, randomization failures, and measures to address these problems.

As in other experimental and observational studies, recruitment of subjects for the study can be an issue when doing field experiments. Especially if the experiment requires subjects' informed consent about the treatment and if the study is about a sensitive or controversial topic, many potential subjects might refuse to participate and undermine random sampling. While general refusal to participate regardless of the specific assignment to an experimental group does not affect internal validity, it can substantially undermine external validity of results. As explained in section 2 of this chapter, the effect of deviations from random sampling on generalizability depends on how systematic refusal is and whether the treatment effect interacts with the selection mechanism. If subjects have a special motivation to participate in the study, e.g. because they are in special need or expect the intervention to be particularly effective, and if this motivation is correlated with the effectiveness of the treatment, then researchers should be careful to transport their findings to other populations.

Besides deviations from random sampling, which are common in all empirical studies, field experiments are particularly prone for randomization failure. Experimental units might refuse to participate in one specific experimental group, might not comply with the treatment assignment, or might drop out of the study in the course of time. In addition, the staff might not administer the treatment according to the experimental protocol. For example, in the Lanarkshire Milk Experiment (Leighton and McKinlay 1930) teachers should give milk to 10,000 randomly selected children over a period of 4 month to investigate effects on physical development, while 10,000 additional randomly selected children not receiving extra milk should serve as the control. However, as highlighted by Student (1930), teachers gave the milk with a higher probability to poorer children undermining random assignment and resulting in superior body weight and body height after the treatment in the control group.

Due to potential deviations from random assignment, it is advisable to conduct randomization checks exploring whether assignment is compromised. These randomization checks rely on the idea that the distribution of key variables in the different experimental groups 
is expected to be balanced if randomization works. However, if the number of observations per group is small, the covariate distributions between the groups can also differ simply by chance. A blocked or stratified randomized design can help here to secure a comparable distribution of covariates across experimental groups (covariate balance), while the literature does not agree on whether researchers should include covariates into the statistical analysis in case of detected imbalances.

Another common reason for unwanted differences between experimental groups is that the staff administrating the treatment does not or cannot comply with the randomization procedure. Implementers might do this on purpose to influence results or to reduce workload (e.g., by treating all persons in an area). Practical constraints such as hard to reach subjects, logistic issues, or shortages of the treatment (e.g., serum for vaccination) might also limit staff's ability and willingness to comply with the assignment as intended by the researchers.

While these reasons can lead to serious deviations from random assignment in practice, one of the most common caveats in field experiments is refusal of experimental units to be assigned to a certain experimental group. In general, such non-compliance occurs, if the actual assignment does not match with the intended assignment. On the one hand, subjects might refuse to be assigned to the treatment group, if the treatment is potentially harmful or is at least perceived by the subjects to be so. On the other, subjects might want to be part of the treatment group, if they think that the withheld intervention is beneficial to them. Unfortunately, not all sorts of non-compliance are obvious to the researcher. Subjects might formally accept the assignment but then might not comply with the requirements and guidelines for their experimental group. In medical research, lacking compliance of patients (e.g., not taking ascribed pills) is a well-known problem but the issue extends to most other forms of field experiments.

Luckily, the experimental toolbox offers remedies to attenuate the problems caused by noncompliance in experiments. In order to get a basic understanding of how these approaches work, it is essential to distinguish different types of compliance and non-compliance. As can be seen in Table 1, subjects can belong to one of four different types; following the terminology proposed by Angrist, Imbens and Rubin (1996) these four types are usually called compliers, always-takers, never-takes, and defiers. Compliers always follow the intended assignment and hence are no cause for trouble. The remaining three types, however, give reason to worry, since they deviate from the intended assignment. Always-takers join the treatment group even if they are assigned to the control group. In contrast, never-takers enter the control group regardless of their assignment and are never treated. Finally, defiers always do exactly the opposite of the 
intended assignment (e.g., because they are notoriously suspicious about their assignment to any experimental group).

Table 1: Different Types of Compliance/Non-compliance

\begin{tabular}{|c|c|c|c|c|}
\hline \multirow{3}{*}{$\begin{array}{c}\text { Researcher } \\
\text { Intended } \\
\text { Assignment }\end{array}$} & \multicolumn{4}{|c|}{ Subjects } \\
\hline & \multicolumn{4}{|c|}{ One-sided Non-compliance } \\
\hline & Compliers & Always-takers & Never-takers & Defiers \\
\hline Treatment group & $\checkmark$ & $\checkmark$ & $x$ & $x$ \\
\hline Control group & $\checkmark$ & $x$ & $\checkmark$ & $x$ \\
\hline
\end{tabular}

Note: $\checkmark$ indicates that subjects comply with the assignment intended by the researcher, $x$ that they do not comply.

While standard statistical tools such as simple comparisons of group means are sufficient to analyse experimental data for compliers, experiments with one- or two-sided non-compliance require special attention and should to be analysed using specific statistical procedures such as those discussed below. Simply using the actual instead of the intended assignment to experimental conditions is a bad idea, since this approach neglects the fact some of the subjects self-selected into or out of the treatment group and do not comply with researchers intention to treat them. Remember that it was the original motivation for conducting experiments in the first place to secure a high degree of internal validity, since the combination of random assignment and treatment manipulation helps to avoid both selection bias and confounding by third variables. Non-compliance undermines random assignment resulting in biased estimates of treatment effects, because in most cases there is no good reason to assume that the four types of compliance/non-compliance do not systematically differ with respect to variables relevant for the outcome and the treatment effect. Simply removing non-compliers from the analysis is also problematic since it leads to biases for similar reasons of non-random assignment and selfselection.

So what to do instead? As a starting point one has to accept that the experiment didn't work as intended for all subjects and, hence, not all observations are equally useful for estimating the treatment effect. As a result, it is usually not possible to provide a correct estimate of the average treatment effect for all experimental units. Although this diagnosis of a broken experiments sounds bad, it is important to keep calm and be aware of three facts: (1) Non-compliance is nothing unusual. It is a frequent phenomenon in field experiments. One might probably even argue that it is the standard case for most field experiment, while a study with $100 \%$ compliers is exceptional. (2) The broken experiment can still provide important and reliable information 
on causal effects of the treatment. (3) Uncovering this information requires adequate statistical analysis.

One well-established statistical approach to deal with always-takers and never-takers is the so-called intention-to-treat (ITT) analysis. The basic idea of the ITT is to use the intended instead of the actual assignment for the group comparison. For example, imagine a scenario with compliers and never-takers. Some subjects assigned to the treatment group switch to the control group, but no subjects assigned to the control group switch to the treatment group (called one-sided non-compliance) $)^{3}$. In this case one would treat never-takers in the ITT analysis as if they had received the treatment. The effect of the ITT is then simply the average difference in the outcomes between experimental units intended to be assigned to the treatment and control group.

This effect of ITT is not identically to the average treatment effect in case of systematic noncompliance, but it is still informative under certain assumptions: ${ }^{4}$ it measures the effect of intended treatment assignment and treatment if taken up by the subjects. In the described scenario with only two types of (non-)compliance - compliers and never-takers - the ITT would provide a lower bound of the average treatment effect. That is the effect of the intervention if all subjects assigned to the treatment group actually receive the treatment. For some policies such an ITT analysis makes a lot of sense, because for many real-world interventions it is realistic to assume that not all subjects eligible for the treatment actually get treated. Some designs - so-called encouragement designs (see also section 4) - actually anticipate and exploit the fact that only some experimental units pick up the treatment and deliberately rely for practical or ethical reasons on an ITT analysis to derive conclusions.

A final note concerns defiers: The ITT analysis is not able to deal with this group of subjects adequately. It is thus advisable to design the study in a way to keep the amount of defiers to a minimum. Convincing subjects about the use of the experiment, providing incentives comply with the assignment, and conducting concealed field experiments are three measures to reach that aim.

\footnotetext{
${ }^{3}$ One-sided compliance occurs if subjects assigned to one experimental group do not adhere to the assignment, while subjects assigned to the other experimental group do. One-sided compliance thus occurs in cases with only never-takers or with only always-takers. If both groups are simultaneously present or if defiers take part in the study, the experiment is said to suffer from two-sided compliance.

${ }^{4}$ For more details on statistical background, the links to the counterfactual framework of causal inference, key identification assumptions, and related estimators see Gerber and Green (2012, Chap. 5 \& 6).
} 


\section{$4 \quad$ Ethical and Practical Aspects}

As explained in the beginning of this chapter, field experiments are staged in the natural environment of experimental units. This implies two important things which will be discussed in more detail in this subsection. First, treatments need to be implemented in this natural environment which raises practical issues such as field access and logistics. Second, experimental interventions in natural contexts have real consequences for human beings making questions about research ethics particularly pressing in field experiments.

On the practical side, access to the field is essential and often only possible if granted by local cooperation partners. For example, Delfgaauw et al. $(2014,2015)$ conducted a field experiment on the effects of prizes for employees on sales in stores of a retail chain. Apparently, such an experiment is only realizable if the management of the company approves or at least tolerates the study. Doing field experiments therefore requires social skills and often involves substantial investments to convince local cooperation partners to support the study. Besides the general issue of gaining access to the field, these third agents also need to be convinced that the core elements of the experiment - treatment manipulation, grouping, and random assignment are essential for the quality of the study and cannot be easily dropped.

This cannot be emphasized enough, because the experimental design in these studies is frequently the object of scepticism and fierce critique for several reasons. First, local cooperation partners are usually not aware of the drawbacks of observational studies and the advantages of field experiments for causal inference. Communicating the core arguments in non-technical terms can help. Second, field experiments are often more expensive and time consuming than similar observational studies. They require additional staff to implement and randomly assign the treatment as well as to monitor processes in the field and convince subjects to participate. All this involves additional administrative and logistical challenges, can interrupt everyday routines, and is an additional burden for staff members that comes on top of their regular tasks and responsibilities. Given that local cooperation partners often do not have an extra budget for these additional efforts it doesn't come as a surprise that they raise the question whether the experiment is really necessary. Third, lacking empirical knowledge about the effect of the treatment is the reason for conducting the experiment. Randomly assigning some units to a potentially harmful intervention and/or excluding subjects in the control group from a potentially beneficial treatment is often criticized by different stakeholders. For this reason, politicians and other cooperation partners originally sympathizing with the experiment might withdraw their initial support in anticipation of public critique. Involved subjects might react 
with refusal to participate and non-compliance. And third parties not directly involved in the study might even engage in purposeful activities to undermine the study and its credibility.

At the core of this conflict is the random assignment of the treatment as it is perceived as unethical and unfair to withhold the intervention from some and to provide it to others simply based on chance. Indeed, sometimes critics touch a sore spot of experiments here, especially if enough resources are available to treat all subjects in an area. For example, it is hard to imagine to withhold an effective drug from a patient if enough resources are available to treat all sick patients. However, the standard case is that resources are limited and do not suffice to treat all. A random mechanism, such as a lottery, isn't such a bad allocation mechanism in face of such constraints and well accepted by many, especially if combined with certain criteria for treatment eligibility such as need-based access.

Moreover, experimentalists have conceived other designs to address critique voiced against random assignment. In the phase-in design all subjects receive the treatment, however at different points in time. For example, in the phase-in design depicted in Table 2, group A receives the treatment first, group B gets treated 6 later, and group $\mathrm{C}$ receives the treatment after 12 months. Hence, different groups can serve as comparison group at different time points. While this still design raises the question of how gets the treatment first, the approach certainly attenuates ethical concerns.

Finally, encouragement designs do not directly assign the treatment, but only encourage subjects by means of an intervention to actually use it, e.g. by distributing monetary vouchers for attending private schools (Angrist et al. 2002) or encouraging people to vote (Green and Gerber 2015). While the treatment "encouragement" is still withheld from part of the subjects allowing to estimate treatment effects, no subject in the treatment group is forced to follow the encouragement and to receive a potentially harmful treatment. In these designs, researchers knowingly accept for ethical or practical reasons that many subjects will not comply with the intended assignment. However, as shown before, encouragement designs can still provide valuable insights using an intention-to-treat analysis.

Table 2: Phase-in design

\begin{tabular}{cccc}
\hline & \multicolumn{3}{c}{ Time in months } \\
\cline { 2 - 4 } Group & $\mathbf{0 - 6}$ & $\mathbf{7 - 1 2}$ & $\mathbf{1 3 - 1 8}$ \\
\hline $\mathrm{A}$ & $\mathrm{T}$ & $\mathrm{T}$ & $\mathrm{T}$ \\
$\mathrm{B}$ & $\mathrm{C}$ & $\mathrm{T}$ & $\mathrm{T}$ \\
$\mathrm{C}$ & $\mathrm{C}$ & $\mathrm{C}$ & $\mathrm{T}$ \\
\hline
\end{tabular}

Note: $\mathrm{T}$ indicates "treated", $\mathrm{C}$ indicates "control" 
The discussion of alternatives to regular random assignment already touched the topic of research ethics when designing and doing field experiments. Concluding this chapter, we will discuss this topic in more detail now. To start with, field experiments underlie the same code of ethics as other types of social science research. However, because field experiments are staged in natural contexts and because researchers "playing god" (Baldassari and Abascal 2017) intervene in these social environments with sometimes serious long-term consequences for experimental units or communities, the issue of research ethics deserves special attention when conducting field experiments. As Gerber and Green (2012) recommend, researchers should try to choose treatments that have positive effects on human lives and should avoid potentially harmful treatments whenever possible. While obvious in some instances, this is easier said than done in other cases: In complex social environments the effects of a treatment are far from clear, negative externalities need to be taken into account, and unanticipated effects (e.g., due to social dynamics or reactivity) can occur (Teele 2014).

Following standard codes of ethics, researchers further need to respect subjects' personal rights, obtain informed consent before the experiment, and debrief them after the study. However, in order to avoid methodological problems - such as reactivity, refusal to participate and non-compliance - researchers do not always follow these guidelines in practice. A classical case in point are techniques of unobtrusive measurement which do not suffer from reactivity and are particularly well-suited for investigating sensitive topics such as crime and deviance. Obviously, in such cases two different goals - personal rights and scientific interest - conflict with each other. Unfortunately, attaining both to the same degree is not always feasible. Since one goal does not always trump the other, it necessary to weigh the costs and benefits of a study regarding both dimensions.

Imagine an unobtrusive field experiment that violates some basic personal rights by employing unobtrusive measures and not informing the subjects about being studied. The experiment also interrupts everyday routines and steals time from the uninformed participants. At the same time the research inflicts no harm on them and generates valuable insights for society. How would you decide in this case? It is hard to provide a general answer because this decision involves normative evaluations which can differ a lot by the specific field of application and by the personal values of the person conducting the cost-benefit analysis. In case of such goal conflicts, we thus highly recommend to consult an ethics committee or institutional review boards (IRB) before running the field experiment and ask for approval of the experiment after weighing costs and benefits. The result of such a process can go in both 
direction. Besides directly approving or rejecting the research, the IRB might also suggest modifications of the design or might only give approval conditional on some requirements. Although this process can consume time and energy, approval by an IRB is important because such ethical oversight reduces the risk for researchers if ethical concerns arise later on or if subjects appeal in court against the study. Moreover, it is worth noting that many journals in the social and behavioural sciences require an IRB approval for publishing field experimental evidence. Certainly, after having designed and conducted a smart field experiment that takes into account all potential pitfalls and provides convincing causal evidence, you want others to read about it. Getting approval of your design by the IRB processes thus is as much part of doing field experiment as conducting the study itself.

\section{Five Lessons from this Chapter}

$\checkmark$ Field experiments rely on the three design elements common to all experiments manipulation, group comparison, and randomization - and, in addition, are staged in a "natural" research setting.

$\checkmark$ Naturalness not only refers to the field setting of the experiment, but to all four UTOS dimensions: units, treatments, outcomes, and settings.

$\checkmark$ In addition to the general strength of experimental designs regarding internal validity, conducting field experiments in "natural" settings offers advantages for generalizing results.

$\checkmark$ The combination of field experimental designs and unobtrusive measurement helps to avoid social desirability.

$\checkmark$ Non-compliance of subjects can be a major challenge for doing field experiments which, however, can be addressed with an intention-to-treat analysis.

\section{References}

Allison, P.D. (2001): Missing Data. Thousand Oaks, CA: Sage

Angrist, J.D., Bettinger, E., Bloom, E., Kremer, M. \& King, E. (2002): The effects of school vouchers on students: Evidence from Colombia. American Economic Review 92: 1535-1558.

Angrist, J.D., Imbens, J.W. \& Rubin, D.B. (1996): Identification of causal effects using instrumental variables. Journal of the American Statistical Association 91: 444-455.

Baldassarri, D. \& Abascal, M. (2017): Field experiments across the social sciences. Annual Review of Sociology 43: 41-73.

Cohn A., Fehr E. \& Maréchal M.A. (2014): Business culture and dishonesty in the banking industry. Nature 516: 86-89.

Cronbach, L.J. (1982): Designing Evaluations of Educational and Social Programs. San Francisco: Jossey Bass. 
Deaton, A. (2010): Instruments, randomization, and learning about development. Journal of Economic Literature 48: 424-455.

Deaton, A. \& Cartwright, N. (2018): Understanding and misunderstanding randomized controlled trials. Social Science and Medicine 210: 2-21.

Delfgaauw, J., Dur, R., Non, A. \& Verbeke, W. (2014): Dynamic incentive effects of relative performance pay: A field experiment. Labour Economics 28: 1-13.

Delfgaauw, J., Dur, R., Non, A. \& Verbeke, W. (2015): The effects of prize spread and noise in elimination tournaments: A natural field experiment. Journal of Labor Economics 33: 521-569.

Diekmann, A., Jungbauer-Gans, M., Krassnig, H. \& Lorenz, S. (1996): Social status and aggression. A field study analyzed by survival analysis. Journal of Social Psychology 136: 761-768.

Dobb, A.N. \& Gross, A.E. (1968): Status of frustrator as an inhibitor of horn-honking responses. Journal of Social Psychology 76: 213-218.

Enders, C.K. (2010): Applied Missing Data Analysis. New York: Guilford Press.

Falk, A. \& Heckman, J.J. (2009): Lab experiments are a major source of knowledge in the social sciences. Science 326: 535-538

Gächter, S. \& Herrmann, B. (2011): The limits of self-governance when cooperators get punished: Experimental evidence from urban and rural Russia. European Economic Review 55: 193-210.

Gerber, A.S. \& Green, D.P. (2012): Field Experiments. Design, Analysis, and Interpretation. New York/London: W.W. Norton \& Company.

Gillespie, G. (1991): Manufacturing Knowledge. A History of the Hawthorne Experiments. Cambridge, MA: Cambridge University Press.

Gneezy, A. (2017): Field experimentation in marketing research. Journal of Marketing Research 54: $140-143$.

Green, D.P. \& Gerber, A.S. (2015): Get Out The Vote: How to Increase Voter Turnout (3 ${ }^{\text {rd }}$ edition). Washington, D.C.: Brookings Institution Press.

Greenberg, D. \& Shroder, M. (2004): The Digest of Social Experiments ( $3^{\text {rd }}$ edition). Washington: urban Institute Press.

Haney, C., Banks, W.C. \& Zimbardo, P.G. (1973): Interpersonal dynamics in a simulated prison. International Journal of Criminology and Penology 1: 69-97.

Harrison, G.W. \& List, J.A. (2004): Field experiments. Journal of Economic Literature 42: 1009-1055.

Heckman, J.J. \& Smith, J.A. (1995): Assessing the case for social experiments. Journal of Economic Perspectives 9: 85-110.

Jann, B. (2009): Sozialer Status und Hup-Verhalten. Ein Feldexperiment zum Zusammenhang zwischen Status und Aggression im Strassenverkehr. In P. Kriwy \& C. Gross (eds.), Klein aber fein! Quantitative empirische Sozialforschung mit kleinen Fallzahlen. Wiesbaden: VS Verlag. Pp. 397-410.

John, P. (2017): Field Experiments in Political Science and Public Policy. New York: Routledge.

Leighton, G. \& McKinlay, P.D. (1930): Milk Consumption and the Growth of School Children. Department of Health for Scotland. Edinburgh and London: Her Majesty's Stationery Office.

Levitt, S.D. \& List, J.A. (2009): Field experiments in economics: The past, the present, and the future. European Economic Review 53: 1-18.

List, J.A. (2011): Why economists should conduct field experiments and 14 tips for pulling one off. Journal of Economic Perspectives 25: 3-16.

Little, R.J.A. \& Rubin, D.B. (2002): Statistical Analysis with Missing Data. New York: Wiley.

Lynch, J.G., Jr. (1999): Theory and external validity. Journal of the Academy of Marketing Science 27: $367-376$.

Lynn, P. (ed.) (2009): Methodology of Longitudinal Surveys. New York: Wiley.

Piza, E. (2018): The crime prevention effect of CCTV in public places: A propensity score analysis. Journal of Crime and Justice 41: 14-30.

Ridgeway, C.L. (2014): Why status matters for inequality. American Sociological Review 70: 1-16.

Roethlisberger, F.J. \& Dickson, W.J. (1939): Management and the Worker: An Account of a Research Program Conducted by the Western Electric Company, Hawthorne Works, Chicago. Cambridge, MA: Harvard University Press. 
Rosenthal, R.W. \& Jacobson, L. (1968): Pygmalion in the Classroom. New York: Holt, Rinehart \& Winston.

Rubin, D.B. (1980): Randomization analysis of experimental data: The Fisher randomization test. Journal of the American Statistical Association 75: 591-593.

Shadish, W.R., Cook, T.D. \& Campbell, D.T. (2002): Experimental and Quasi-experimental Designs for Generalized Causal Inference. Boston, MA: Houghton, Mifflin and Company.

Student (1931): The Lanarkshire milk experiment. Biometrika 23: 398-406.

Teele, D.L. (ed.) (2014): Field Experiments and Their Critics: Essays on the Uses and Abuses of Experimentation in the Social Sciences. New Haven/London: Yale University Press.

Wolbring, T., Bozoyan, C. \& Langner, D. (2013): "Walk left, stand right!" A field experiment on the enforcement of informal norms on escalators. Zeitschrift für Soziologie 42: 239-258. 